\title{
The mysterious eruption of V838 Mon
}

\author{
U. Munari ${ }^{1}$, A. Henden ${ }^{2}$, S. Kiyota ${ }^{3}$, D. Laney ${ }^{4}$, F. Marang ${ }^{4}$, T. Zwitter ${ }^{5}$, R. L. M. Corradi ${ }^{6}$, S. Desidera ${ }^{1}$,

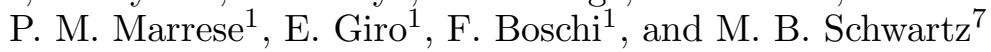 \\ 1 INAF-Osservatorio Astronomico di Padova, Sede di Asiago, 36012 Asiago (VI), Italy \\ 2 Univ. Space Research Ass./U. S. Naval Observatory, PO Box 1149, Flagstaff AZ 86002-1149, USA \\ 3 VSOLJ, 1-401-810 Azuma, Tsukuba 305-0031 Japan \\ 4 South African Astronomical Observatory, PO Box 9, Observatory 7935, South Africa \\ 5 University of Ljubljana, Department of Physics, Jadranska 19, 1000 Ljubljana, Slovenia \\ 6 Isaac Newton Group of Telescopes, Apartado de Correos 321, 38700 Santa Cruz de La Palma, Canarias, Spain \\ 7 Tenagra Observatory, HC2 Box 292 Nogales, AZ 85621, USA
}

Received 12 April 2002 / Accepted 16 May 2002

\begin{abstract}
V838 Mon is marking one of the most mysterious stellar outbursts on record. The spectral energy distribution of the progenitor resembles an under-luminous $\mathrm{F}$ main sequence star (at $V=15.6 \mathrm{mag}$ ), that erupted into a cool supergiant following a complex and multi-maxima lightcurve (peaking at $V=6.7 \mathrm{mag}$ ). The outburst spectrum show BaII, LiI and lines of several $s$-elements, with wide P-Cyg profiles and a moderate and retracing emission in the Balmer lines. A light-echo discovered expanding around the object helped to constrain the distance ( $d=790 \pm 30 \mathrm{pc}$ ), providing $M_{V}=+4.45$ in quiescence and $M_{V}=-4.35$ at optical maximum (somewhat dependent on the still uncertain $E_{B-V}=0.5$ reddening). The general outburst trend is toward lower temperatures and larger luminosities, and continuing so at the time of writing. The object properties conflict with a classification within already existing categories: the progenitor was not on a post-AGB track and thus the similarities with the born-again AGB stars FG Sge, V605 Aql and Sakurai's object are limited to the cool giant spectrum at maximum; the cool spectrum, the moderate wind velocity $\left(500 \mathrm{~km} \mathrm{~s}^{-1}\right.$ and progressively reducing) and the monotonic decreasing of the low ionization condition argues against a classical nova scenario. The closest similarity is with a star that erupted into an M-type supergiant discovered in M 31 by Rich et al. (1989), that became however much brighter by peaking at $M_{V}=-9.95$, and with V4332 Sgr that too erupted into an M-type giant (Martini et al. 1999) and that attained a lower luminosity, closer to that of V838 Mon. M 31-RedVar, V4332 Sgr and V838 Mon could be all manifestations of a new class of astronomical objects.
\end{abstract}

Key words. stars: supergiants - stars: novae - stars: individual: V838 Mon - stars: mass-loss - ISM: jets and outflows

\section{Introduction}

The previously unnoticed, highly peculiar object V838 Mon was discovered in outburst by Brown (2002) on January 6 . The complex lightcurve, cool colors at maximum (in spite of a $\triangle m=9$ mag amplitude), strong mass loss and a spectrum rich in $s$-process elements, a peak $V=6.7 \mathrm{mag}$ and a favorable position on the celestial equator favored massive world-wide interest and an observational effort that has so far resulted in 23 IAU Circulars. In this Letter we present and discuss our astrometric, photometric, spectroscopic, imaging and polarimetric observations. The basic properties of V838 Mon in quiescence and outburst are derived, and its nature outlined. Only a fast and preliminary analysis of the large amount of gathered information will be possible in this Letter. A more complete data analysis will be performed elsewhere.

We obtained CCD $U B V R_{\mathrm{c}} I_{\mathrm{c}}$ photometry of V838 Mon with the USNO $1 \mathrm{~m}$ telescope in Flagstaff and a privately

Send offprint requests to: U. Munari,

e-mail: munari@pd.astro.it operated $25 \mathrm{~cm}$ telescope in Tsukuba, Japan, against a photometric sequence that we calibrated with respect to Landolt's equatorial standards (sequence and identification chart available via http://ulisse.pd.astro.it/ v838_Mon/). The photometry is reported in Tables 1 and 2 (with errors a few units of the last decimal figure). $U$ band imaging has also been provided by the $0.81 \mathrm{~m}$ telescope of Tanagra Obs. (Arizona) and the NOT $2.5 \mathrm{~m}$ and WHT $4.2 \mathrm{~m}$ telescopes in La Palma (Canary Islands). JHKL photometry (on the Carter system) of V838 Mon has been secured with the $0.75-\mathrm{m}$ telescope at SAAO equipped with the IRP Mk II photometer. The magnitudes are listed in Table 3, with errors around 0.01 mag. High resolution spectra of V838 Mon have been regularly obtained since outburst onset with the Echelle+CCD spectrograph of the $1.82 \mathrm{~m}$ Asiago telescope, covering the range 4600$9800 \AA$ at 18500 resolving power. Finally, medium resolution spectroscopy (res.power 6000) has been obtained with AFOSC at the $1.82 \mathrm{~m}$ Asiago telescope over the $3900-7700 \AA$ range, as well as spectropolarimetry (2 and $4 \AA /$ pix $)$ and polarimetric imaging $\left(U B V R_{\mathrm{c}} I_{\mathrm{c}}\right)$ on several nights each month since the onset of the outburst. 


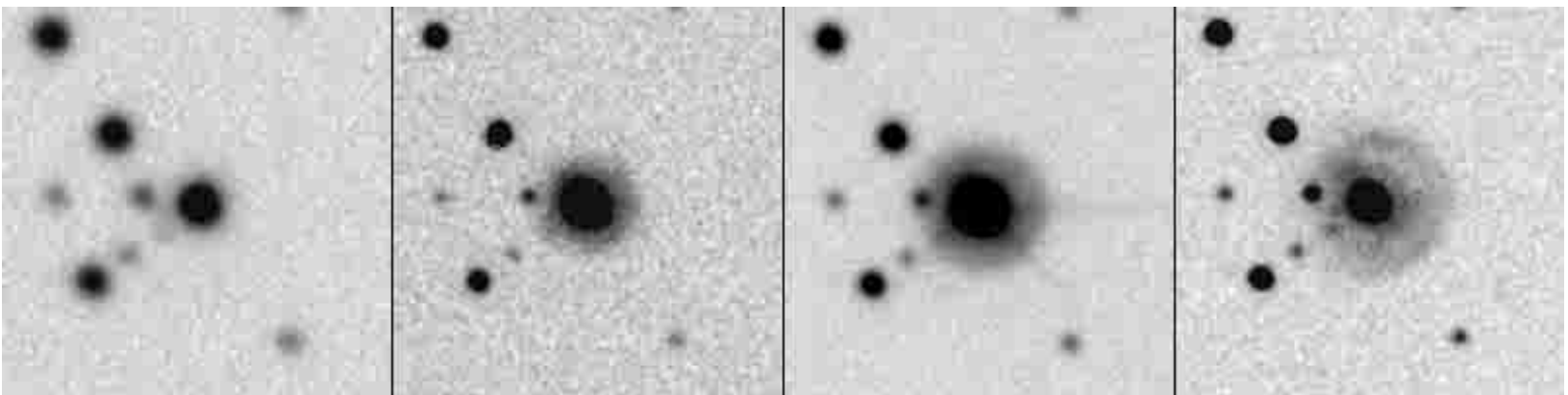

Fig. 1. Expansion of the light-echo around V838 Mon, revealing a previously invisible ring of circumstellar material. $U$ band $67 \times 67$ arcsec images obtained with the USNO $1 \mathrm{~m}$ telescope (North to top, East to the left). Dates (seeing in arcsec, $U$ mag of central V838 Mon) from left to right: Jan. $13\left(3.2^{\prime \prime}, U=13.33\right)$, Feb. $27\left(2.3^{\prime \prime}, U=12.05\right)$, March $10\left(2.5^{\prime \prime}, U=10.62\right)$ and March $27\left(2.2^{\prime \prime}, U=12.28\right)$.

Table 1. USNO UBVRI photometry of V838 Mon in outburst.

\begin{tabular}{cccccc}
\hline \hline HJD & $V$ & $B-V$ & $U-B$ & $V-R_{\mathrm{c}}$ & $R-I_{\mathrm{c}}$ \\
\hline 2452285.7231 & 9.977 & 1.756 & 1.917 & 0.959 & 0.942 \\
2452287.8159 & 9.881 & 1.687 & 1.760 & 0.925 & 0.889 \\
2452288.8061 & 9.896 & 1.681 & 1.771 & 0.938 & 0.873 \\
2452309.6288 & 7.473 & 1.130 & 0.261 & 0.717 & 0.804 \\
2452312.6482 & 6.926 & 0.981 & 0.239 & & \\
2452313.6567 & 7.039 & 1.107 & 0.398 & & \\
2452314.7245 & 7.258 & 1.128 & 0.508 & 0.699 & 0.759 \\
2452315.6028 & 7.420 & 1.156 & 0.570 & 0.703 & 0.795 \\
2452316.6553 & 7.614 & 1.191 & 0.662 & 0.721 & 0.731 \\
2452317.6014 & 7.727 & 1.254 & 0.689 & 0.761 & 0.806 \\
2452321.6405 & 7.913 & 1.494 & & & \\
2452322.7572 & 7.896 & 1.654 & 1.025 & 0.942 & 0.862 \\
2452330.6609 & 8.124 & 2.016 & 1.503 & 1.079 & 1.037 \\
2452332.6254 & 8.186 & 2.137 & 1.726 & 1.169 & 0.987 \\
2452333.6359 & 8.140 & 2.148 & 1.764 & 1.150 & 1.002 \\
2452337.6832 & 7.624 & 1.811 & 1.324 & 0.997 & 0.962 \\
2452338.6850 & 7.506 & 1.753 & 1.301 & 0.976 & 0.901 \\
2452342.6248 & 7.205 & 1.781 & 1.539 & 0.996 & 0.906 \\
2452343.6502 & 7.195 & 1.790 & 1.631 & 0.974 & 0.935 \\
2452344.6465 & 7.164 & 1.785 & 1.661 & 0.978 & 0.900 \\
2452360.7085 & 7.622 & 2.366 & 2.295 & & \\
2452365.6932 & 7.735 & 2.520 & 2.771 & 1.360 & 1.053 \\
2452369.6217 & 7.943 & 2.595 & 2.590 & & \\
2452373.6287 & 8.254 & 2.635 & 2.555 & 1.418 & 1.315 \\
\hline & & & & &
\end{tabular}

Table 2. Tsukuba BVI photometry of V838 Mon in outburst.

\begin{tabular}{crrccccc}
\hline \hline HJD & \multicolumn{1}{c}{$B$} & \multicolumn{1}{c}{$V$} & \multicolumn{1}{c}{$I_{\mathrm{c}}$} & HJD & $B$ & $V$ & $I_{\mathrm{c}}$ \\
\hline 283.998 & 12.16 & 10.10 & 8.28 & 312.902 & 8.09 & 6.80 & 5.38 \\
285.092 & 11.95 & 9.93 & 8.03 & 316.030 & 8.79 & 7.44 & 5.94 \\
286.023 & 11.77 & 9.79 & 7.93 & 317.040 & 8.95 & 7.61 & 6.06 \\
287.098 & 11.77 & 9.79 & 7.96 & 317.956 & 9.09 & 7.66 & 6.06 \\
290.035 & 11.76 & 9.83 & 7.98 & 324.025 & 9.72 & 7.83 & 5.93 \\
292.952 & 11.95 & 9.95 & 8.01 & 324.959 & 9.77 & 7.83 & 5.93 \\
293.984 & 12.06 & 10.01 & 8.06 & 325.940 & 9.88 & 7.88 & 5.98 \\
297.060 & 12.06 & 10.03 & 8.08 & 337.927 & 9.44 & 7.54 & 5.61 \\
298.005 & 12.06 & 10.02 & 8.08 & 341.056 & 9.16 & 7.12 & 5.26 \\
299.009 & 12.14 & 10.07 & 8.15 & 341.949 & 9.01 & 6.95 & 5.16 \\
300.000 & 12.19 & 10.12 & 8.13 & 343.044 & 8.91 & 7.02 & 5.14 \\
301.931 & 12.35 & 10.25 & 8.22 & 343.931 & 8.96 & 6.94 & 5.14 \\
304.011 & 12.64 & 10.48 & 8.39 & 344.980 & 9.19 & 6.99 & 5.13 \\
304.938 & 12.73 & 10.56 & 8.44 & 346.950 & 9.17 & 7.09 & 5.20 \\
306.987 & 12.85 & 10.67 & 8.51 & 349.007 & 9.45 & 7.25 & 5.28 \\
309.904 & 8.55 & 7.29 & 5.76 & 350.024 & 9.57 & 7.26 & 5.24 \\
311.906 & 7.99 & 6.66 & 5.24 & 354.011 & 9.84 & 7.39 & 5.26 \\
\hline
\end{tabular}

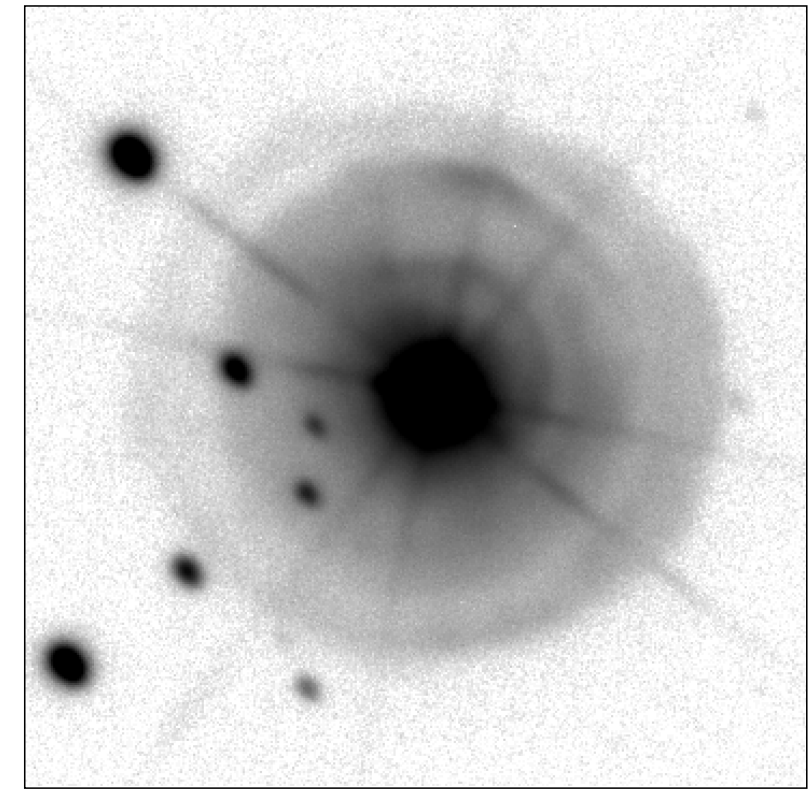

Fig. 2. A median averaged combination of seven 30 -s $U$-band images obtained with the $4.2 \mathrm{~m}$ WHT telescope on March 28, 2002 showing the light-echo (image size 40 arcsec; North to top, East to the left; seeing $0.9 \operatorname{arcsec}, U=12.65 \mathrm{mag}$ for central V838 Mon).

\section{The light-echo and distance to V838 Mon}

A nebula around V838 Mon has been discovered and seen to "expand" with time on broad-band monitoring images obtained with the USNO $1 \mathrm{~m}$ telescope, a sample of which are shown in Fig. 1. The nebula is best seen in $U$ band and it is progressively less evident at longer wavelengths, a pattern typical of light scattering. We interpret the expanding nebula as a light echo of the outburst produced by circumstellar material lost by the progenitor. The nebula is just reflecting light from the central star and it is not self-emitting, because [OIII] imaging and long slit spectroscopy failed to reveal emission lines from it.

The light-echo expansion rate is $0.44 \pm$ 0.017 arcsec day $^{-1}$ in diameter, reaching 30 arcsec by early April on the USNO $1 \mathrm{~m} U$ images used to monitor its development. The light producing the echo is from the second, brighter maximum in the lightcurve (see Sect. 5). Assuming a spherical symmetric distribution 
Table 3. SAAO JHKL photometry of V838 Mon in outburst.

\begin{tabular}{ccccc}
\hline \hline HJD & $J$ & $H$ & $K$ & $L$ \\
\hline 2452285.442 & 6.828 & 6.202 & 5.971 & 5.522 \\
2452309.284 & 4.947 & 4.369 & 4.041 & 3.654 \\
2452309.502 & 4.905 & 4.331 & 4.005 & 3.600 \\
2452311.278 & 4.383 & 3.834 & 3.512 & 3.132 \\
2452313.288 & 4.495 & 3.963 & 3.645 & 3.242 \\
2452314.291 & 4.712 & 4.178 & 3.868 & 3.475 \\
2452315.292 & 4.881 & 4.364 & 4.071 & 3.727 \\
2452316.412 & 5.007 & 4.475 & 4.190 & 3.875 \\
2452317.384 & 5.061 & 4.509 & 4.230 & 3.885 \\
2452318.356 & 5.053 & 4.487 & 4.173 & 3.885 \\
2452319.349 & 5.037 & 4.408 & 4.116 & 3.825 \\
2452320.429 & 4.951 & 4.323 & 4.030 & 3.688 \\
2452321.363 & 4.919 & 4.259 & 3.971 & 3.682 \\
2452323.356 & 4.803 & 4.136 & 3.837 & 3.572 \\
2452324.336 & 4.762 & 4.086 & 3.774 & 3.506 \\
\hline
\end{tabular}

of the circumstellar dust, the expansion rate sets the distance of V838 Mon to $790 \pm 30$ pc. A higher resolution image in $U$ band obtained with the WHT $4.2 \mathrm{~m}$ telescope at La Palma (Canary Islands) for March 28 is presented in Fig. 2. This much finer image does not support our first impression (Henden et al. 2002) of a central hole in the nebula based on the USNO $1 \mathrm{~m}$ telescope lower resolution images and the assumption that the scattered light was from the first maximum in the lightcurve. The outburst light sweeping through the circumstellar material allows us to read the recent mass loss history of the AGB progenitor: assuming a typical $15 \mathrm{~km} \mathrm{~s}^{-1}$ velocity for its wind, the light-echo has reached by early April material lost $\sim 3750$ years ago. Some circularly symmetric brightness enhancements are evident, reminiscent of similar structure seen in the surface brightness distribution of several planetary nebulae (cf. Balick et al. 2001, their Fig. 1), usually interpreted as variations in the progenitor AGB mass loss rate. Their angular separation indicate a $\sim 1200$ year recurrence time.

Could the light-echo be originating instead in a slab of interstellar dust? We believe not for three independent reasons. First, given the perfect round shape of the echo, the slab should be flat and perpendicular to the line of sight, i.e. perpendicular to the galactic plane and therefore hardly a stable dynamical condition. Second, the concentric structures seen in better detail in Fig. 2 do not expand with the light-echo and instead seem to remain fixed in space. They are therefore real density structures. The very low probability that such concentric structures develop in the interstellar space and that they also lie perfectly aligned with the line of sight to V838 Mon argue strongly in favor of a circumstellar origin of the lightecho. Finally, V838 Mon progenitor was detected by IRAS (source 07015-0346) in the 60 and $100 \mu \mathrm{m}$ bands with fluxes of 1.4 and 4.6 Jansky $( \pm 10 \%)$, respectively, indicating dust emission at low temperature.

\section{3 . The reddening and polarization}

Zwitter \& Munari (2002) on high resolution spectra taken in January, identified narrow components of NaI and KI superimposed on the wider P-Cyg stellar profiles that if interpreted as interstellar would have consistently indicated a $E_{B-V}=0.80 \pm 0.05$ reddening when their equivalent width is compared with the Munari \& Zwitter (1997) calibration. Figure 3 shows the narrow components as they appeared in late March. On the Neckel \& Klare (1980) extinction maps for the V838 Mon region, an interstellar $E_{B-V} \sim 0.25$ is reached at the 790 pc distance to the object, while $E_{B-V}=0.80$ pertains to distances larger than $3 \mathrm{kpc}$. Therefore, contribution from circumstellar material must be invoked to explain the intensity of narrow $\mathrm{NaI}$ and KI components. How much this circumstellar material also contributes to the reddening is however unknown because, for example, the dust-to-gas ratio could largely differ from the typical interstellar value or the reddening law be different from the standard $R_{V}=3.1$ one.

The reddening affecting V838 Mon probably lies between a minimum $E_{B-V} \sim 0.25$ implied by the distance and a maximum that could be identified with the $E_{B-V}=0.80$ derived from the intensity of narrow $\mathrm{NaI}$ and KI components. A midpoint $E_{B-V}=0.5$ value will be adopted in the rest of this Letter. $E_{B-V}=0.5$ is supported by polarimetry. We have repeatedly measured during February and March the polarization of V838 Mon both in $U B V R_{\mathrm{c}} I_{\mathrm{c}}$ bands and over 4400-7900 $\AA$ medium resolution spectra with AFOSC in polarimetric mode attached to the Asiago $1.82 \mathrm{~m}$ telescope. The data indicate a polarization constant in time, with a wavelength slope characteristic of an interstellar origin (Serkowski's law), amounting to $2.6 \%$ at $5500 \AA$ at a position angle $150^{\circ} \pm 2$. Adopting the average relation $p(\%, V) / E(B-V)=5.0$ between polarization and reddening found by Serkowski et al. (1975), the observed $p=2.6 \%$ corresponds to $E_{B-V}=0.52$.

\section{The progenitor}

An accurate astrometric position for V838 Mon in outburst has been obtained from USNO $1 \mathrm{~m}$ images (linked to the USNO-A2.0 local grid). It allows an identification of the progenitor with an anonymous $V \sim 15.6$ mag star that has positional measurements in both the USNO A2.0 and 2MASS catalogues:

$\begin{array}{cccl}\alpha_{\mathrm{J} 2000} & \delta_{\mathrm{J} 2000} & \text { epoch } & \\ 07: 04: 04.81 & -03: 50: 50.9 & 2002 & \text { ours } \\ 07: 04: 04.82 & -03: 50: 50.5 & 1997 & \text { 2MASS } \\ 07: 04: 04.85 & -03: 50: 51.1 & 1953 & \text { POSS-I }\end{array}$

The marginal or null proper motion supports a low space velocity of the progenitor, and thus a probable partnership with galactic disk stars (a $100 \mathrm{~km} \mathrm{~s}^{-1}$ transverse velocity at $790 \mathrm{pc}$ corresponds to a 1.3 arcsec displacement during the time elapsed between POSS-I and our observations). At galactic coordinates $l=217.80 b=+1.05$, the height over the galactic plane is just $z=13 \mathrm{pc}$, again supporting a link to Pop I stars. The radial velocity of the progenitor is unknown, and values derived from absorption lines in outburst are severely affected by the PCyg profiles of the lines (even using LaII lines that rank 


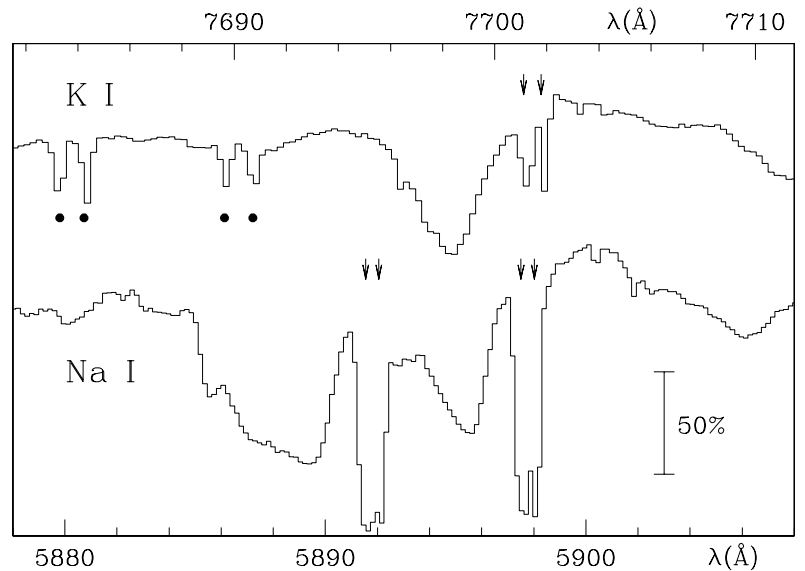

Fig. 3. The spectrum of V838 Mon around NaI and KI lines for March 29, 2002. The dots mark telluric absorptions, the arrows the narrow components discussed in the text. The wide absorptions are the stellar NaI and KI profiles. The bar marks the intensity scale with respect to the normalized continuum.

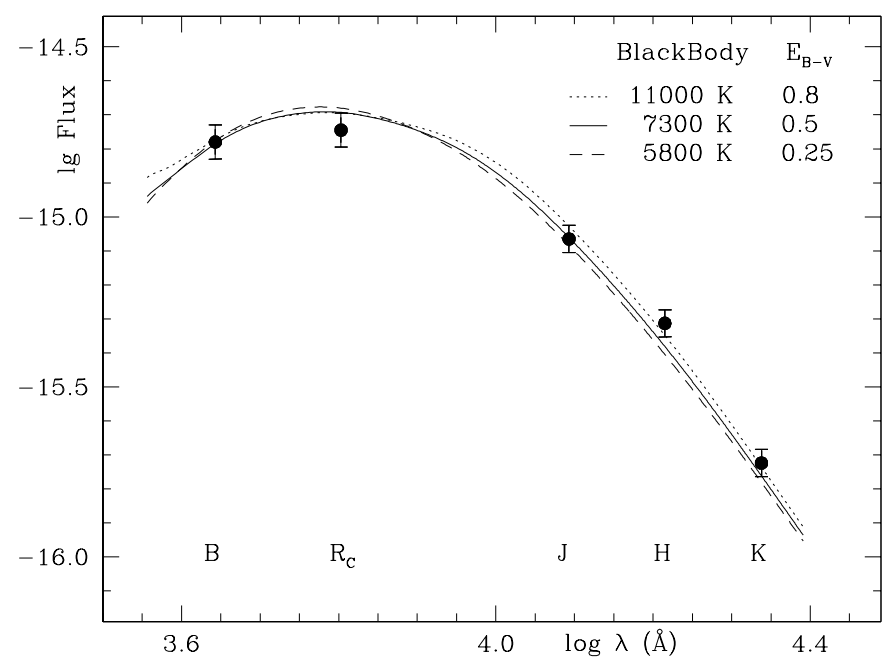

Fig. 4. Spectral energy distribution of V838 Mon in quiescence, for the adopted $E_{B-V}=0.5$, and the extrema of the range of possible values discussed in Sect. 3

among the sharper ones with marginal emission components, the mean heliocentric velocity for late January was $+54.4 \pm 0.8$, for late February $-84.4 \pm 0.2$ and for late March $\left.-57.5 \pm 0.5 \mathrm{~km} \mathrm{~s}^{-1}\right)$.

The brightness of the progenitor has been measured on Palomar and SERC plates by comparison with the photometric sequence we have calibrated:

$\begin{array}{cccccc}\text { Plate } & \text { Date } & \text { UT } & \text { band } & \text { mag } & \\ \text { so0662 } & 1953.0445 & 06.42 & B & 16.00 & \text { POSS-I } \\ \text { se0662 } & 1953.0445 & 07.52 & R_{\mathrm{c}} & 15.35 & " \\ \text { sb0772 } & 1983.0431 & 11.90 & B & 16.10 & \text { SERC } \\ \text { sr0772 } & 1989.1732 & 10.65 & R_{\mathrm{c}} & 15.30 & "\end{array}$

The data support little or no variability of V838 Mon in quiescence during the last half century. They can be therefore safely combined with the near-IR 2MASS measurements $(J=13.91, H=13.48$ and $K=13.35$ obtained in 1997) to derive the spectral energy distribution

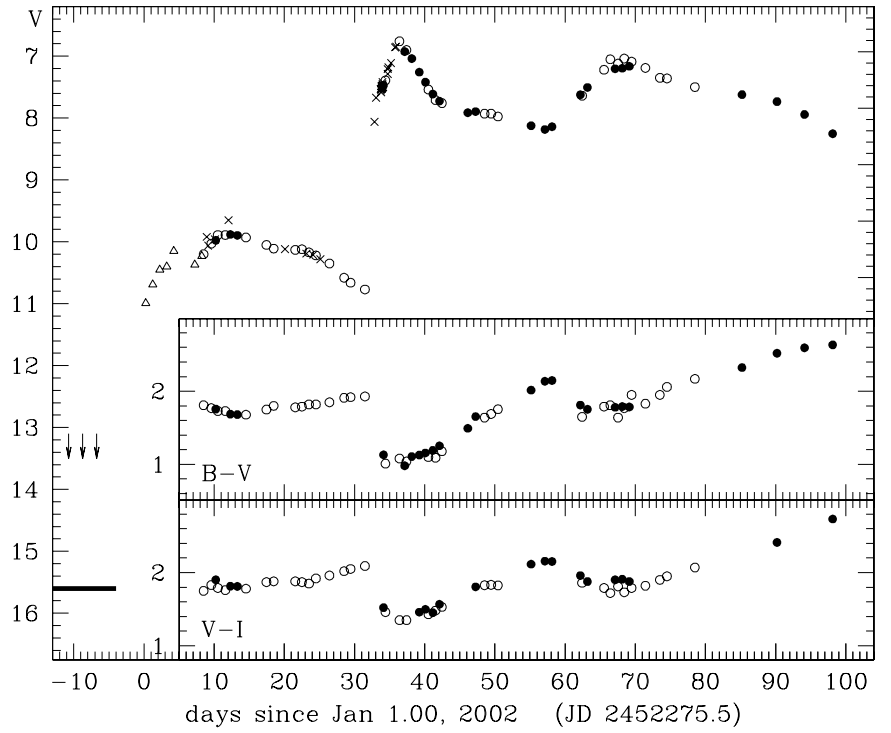

Fig. 5. $V, B-V$ and $V-I_{\mathrm{c}}$ lightcurves of the outburst of V838 Mon. Dots mark data from Table 1, open circles from Table 2. Crosses and open triangles are values from various IAUC and VSNET circulars (mainly from SAAO, D. West, P. Sobotka, L. Smelcer, F. Lomoz and J. Bedient). Arrows mark "fainter than" conditions from IAUC 7785, and the solid line indicates the quiescence brightness.

of the progenitor, which is done in Fig. 4. Even the maximum allowed temperature in quiescence $(11000 \mathrm{~K})$ is not enough to ionize the circumstellar material that produced the light-echo, and in fact a planetary nebula was not observed in quiescence. It is worth to note that the galactic equator has been surveyed several times from both hemispheres in search for emission line objects, and the progenitor of V838 Mon has never been logged in as an emission source (in spite of the favorable magnitude). Several emission line objects have been discovered by such surveys in the region (for example AS 143 lies just 16 arcmin from V838 Mon).

At $d=790 \mathrm{pc}, E_{B-V}=0.5$ and $M_{V}=+4.46$, the best fitting $7300 \mathrm{~K}$ blackbody corresponds to a progenitor of radius of $R \sim 0.8 R_{\odot}$ and $L \sim 1.6 L_{\odot}, 4.5 \times$ less luminous (1.63 mag) than a corresponding F0 V main sequence star (cf. Fig. 8).

\section{The outburst}

V838 Mon went into outburst around the beginning of 2002 , with a fast rise to maximum (a week earlier it was still $V \geq 13.5 \mathrm{mag}$ ). The lightcurve up to early April is presented in Fig. 5. It is characterized by a complex and rarely seen behavior.

A first maximum was reached by day +10 (see abscissae scale in Fig. 5) when the continuum energy distribution was characterized by a temperature of $4150 \mathrm{~K}$ (see dotted line in Fig. 6), a second maximum at $+37^{\mathrm{d}}$ peaked at $5200 \mathrm{~K}$ (solid line in Fig. 6) and a third one at $+68^{\mathrm{d}}$ reached $4600 \mathrm{~K}$. Decline from maxima are marked by monotonic cooling, with the last one taking V838 Mon to $3400 \mathrm{~K}$ by day $+98^{\mathrm{d}}$ (last point in Fig. 5 ). 


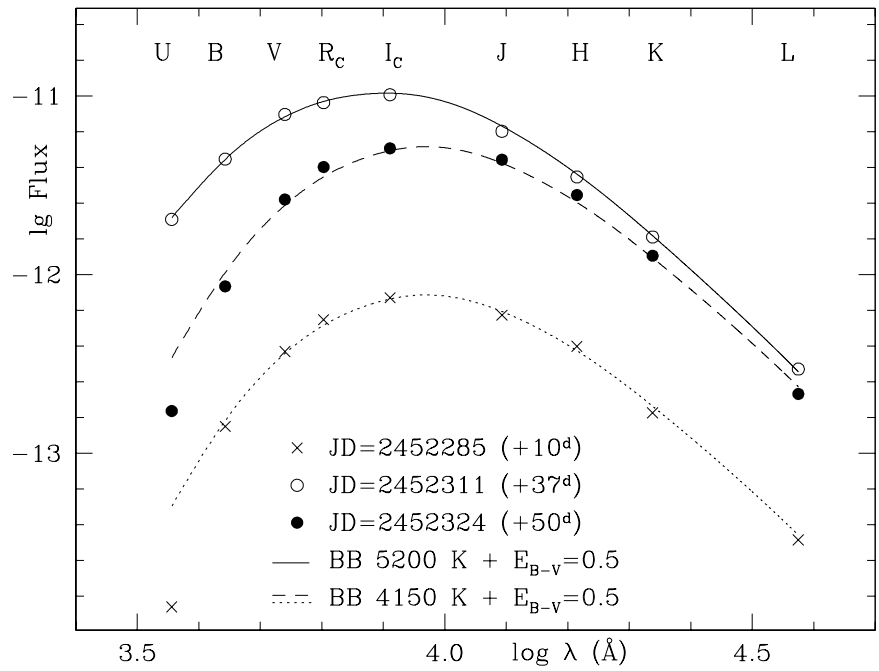

Fig. 6. $U B V R_{\mathrm{c}} I_{\mathrm{c}} J H K L$ energy distribution of V838 Mon in outburst at three dates: first maximum, second maximum and mid plateau (data from Tables 1 and 3).

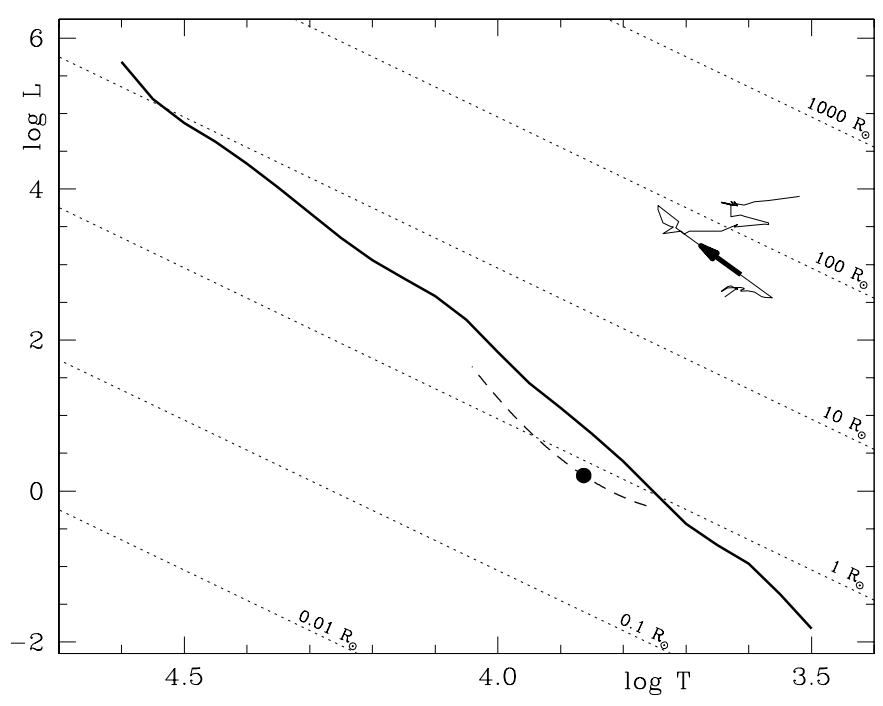

Fig. 7. V838 Mon on the $\log L-\log T$ digram. The diagonal thick line is the Main Sequence (O5 to M5, from Drilling $\&$ Landolt 2000). The solid circle gives the progenitor position and the curve traces the outburst path (from Jan. 11 to Apr. 1, direction arrowed) for the adopted $d=790 \mathrm{pc}$ and $E_{B-V}=0.5$. The range of locations for the progenitor (and equivalent displacements for the outburst path) are indicated by the dashed line (extrema at $d=790 \mathrm{pc}, E_{B-V}=0.25$ and $\left.d=8 \mathrm{kpc}, E_{B-V}=0.80\right)$.

The outburst path on the $\log L-\log T$ diagram is presented in Fig. 7. It can be best described as a trend (with interruptions) toward the upper right corner of the diagram, thus toward progressively cooler surface temperatures and larger radii and greater luminosities.

The spectral appearance of V838 Mon in outburst matches the cool photospheric temperatures indicated by the $U B V R I$ and $J H K L$ photometry. Portions of high resolution sample spectra obtained with the Asiago Echelle spectrograph are presented in Fig. 8. The photospheric temperature at the time these were taken was pretty similar, the $V-I$ index for the three dates being $+2.00,+2.14$ and +2.22 , respectively. The differences seen in the intensity and profiles of the lines is therefore mainly affected by changing wind structure and velocity, as well as lowering of surface gravity (expanding radius).

Late January spectra (toward the end of the decline from first maximum) are characterized by wide P-Cyg profiles of species with low excitation potential (E.P.). In particular CaII, BaII, NaI and LiI lines show remarkably similar profiles and $-500 \mathrm{~km} \mathrm{~s}^{-1}$ terminal velocities. Higher E.P. lines (like those of hydrogen and some FeI multiplets) show instead almost pure absorptions, with narrow and Gaussian-like profiles resembling those expected in a normal cool giant. In late February spectra the P-Cyg terminal velocities of low E.P. species reduce (to $-380 \mathrm{~km} \mathrm{~s}^{-1}$ ) and those of higher E.P. widen, with all lines evolving toward more homogeneous widths and shapes. The reduction of P-Cyg terminal velocities for low E.P. species continued in late March spectra $\left(-280 \mathrm{~km} \mathrm{~s}^{-1}\right)$, with an overall increase in the number and intensity of normal absorption lines. Balmer lines developed an emission component around the second maximum, and have been declining since then.

No chemical abundance analysis will be attempted here, however an eye inspection of the high resolution spectra reveals numerous and strong absorptions from $s$-elements, with marked BaII and a LiI stronger than in the FG Sge and Sakurai's object spectra presented by Kipper (2001).

\section{The nature of $\mathrm{V} 838$ Mon}

The nature of V838 Mon is best described as a mysterious one. It is standard in such cases to state that "more observations are needed", which is certainly true for V838 Mon. Four key tasks for follow-up investigations seem to be: $(a)$ to better address the nature of the progenitor in quiescence by reconstructing its photometric history from plate archives around the globe (using the deep $U B V R I$ comparison sequence available from http://ulisse.pd.astro.it/V838_Mon/), (b) to firmly prove (or disprove) the origin of the light-echo within a circumstellar nebula, which would accurately set the distance, by coronographic observations at widely different wavelengths and epochs and great spatial resolution, $(c)$ to derive an accurate estimate for the interstellar and the circumstellar amount of reddening and extinction (including accurate reddening estimates for many field stars spread over a great range of distances along the line of sight to V838 Mon), (d) when the spectrum will be less perturbed by the wide $\mathrm{P}$-Cyg profiles now in place and it will better resemble a classical photosphere, to perform an accurate chemical abundance analysis as a clue to the outburst causes (like a born-again-AGB scenario and the dredgedup material it implies).

We conclude this Letter with brief considerations about possible scenario interpretations for V838 Mon and its outburst that will be refined in follow-up investigations. 


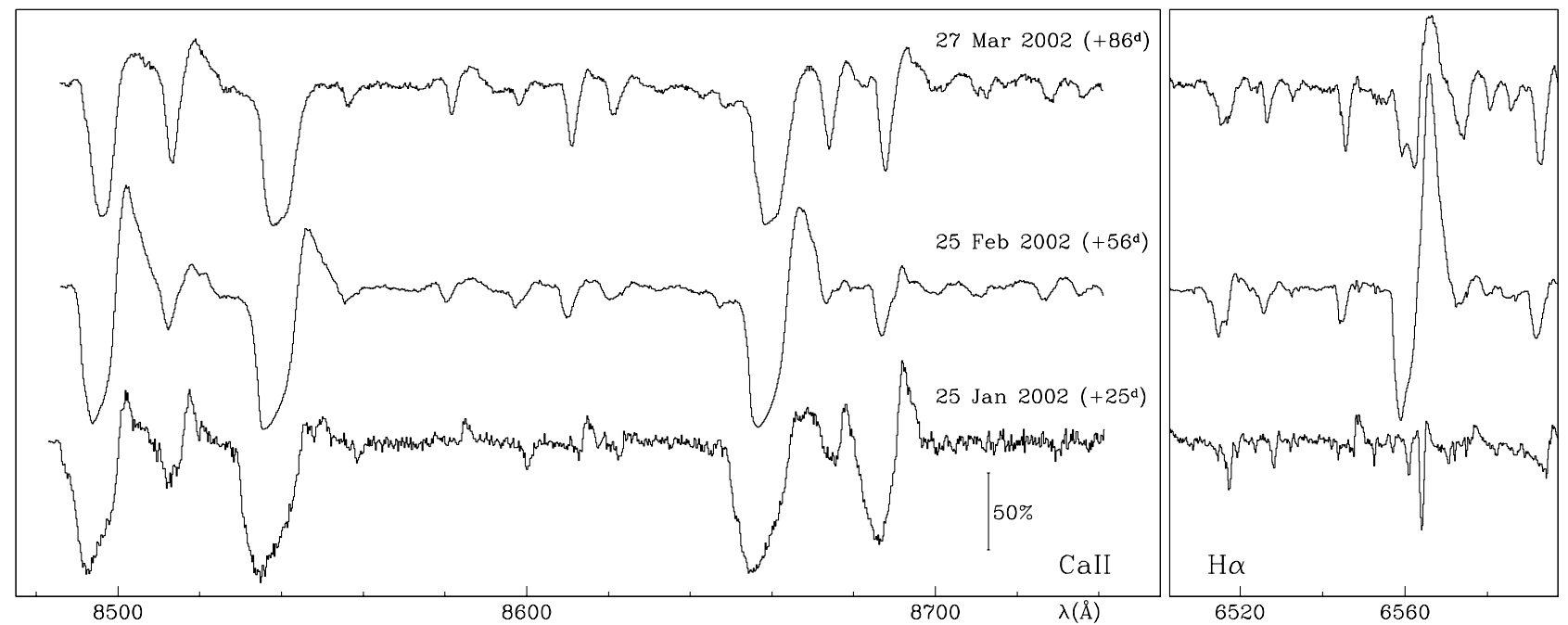

Fig. 8. Small sections of sample Asiago Echelle spectra to document the evolution around the near-IR Calcium triplet and H $\alpha$. The bar marks the intensity scale with respect to the normalized continuum.

A classical nova? The main similarity resides in the rapid rise from quiescence to the first maximum, while several counter-arguments hold strong, such as the lack of variability and emission lines from the probable cataclysmic variable precursor. Also the progenitor spectral energy distribution (an under-luminous $\mathrm{F}$ main sequence star) is quite strange for a $\mathrm{CV}$, and the ejection velocities (not exceeding $500 \mathrm{~km} \mathrm{~s}^{-1}$ ) are low for a classical nova. The very slow evolution could be interpreted within a classical nova framework as an indication for a small mass of the accreting WD, and in turn a small mass of the progenitor, which is however in contrast with the indication of a partnership with the young galactic disk population.

$A$ born-again $A G B$ ? Post-AGB stars on their leftward motion on the HR diagram to become the central stars of planetary nebulae can experience a last helium flash that pushes them back toward the region occupied by AGB stars. Known examples are FG Sge, V605 Aql and Sakurai's object. This is an attractive scenario because V838 Mon in outburst looks much like a cool AGB with a surface rich in dredged-up barium, lithium, $s$-elements, and the circumstellar material producing the light-echo agrees with a recent phase of heavy mass loss at the tip of the AGB (it did not show up as a PN in quiescence - as it was the case for FG Sge, V605 Aql and Sakurai's object - because the precursor was not hot enough to ionize it). However, the progenitor appears severely under-luminous for the standard theoretical tracks of post-AGB stars on the HR diagram (cf. Blöcker \& Schönberner 1997), and the rise to maximum is too fast in comparison with the evolution of outburst of known cases.

A M 31-RedVar or V4332 Sgr analogue? In 1989 an erupting star in the Andromeda Galaxy (M 31) developed a M-type cool supergiant spectrum at maximum, with pronounced P-Cyg profiles and Balmer lines in emission and peaked to $M_{V}=-9.95$ (Rich et al. 1989; Mould et al. 1990). The progenitor was too faint to be identified and the event has been modeled by Iben \& Tutukov (1992) in terms of a cool WD accreting at a very low rate from a companion and under such circumstances the entire WD could experience a thermonuclear runaway. A similar type of eruption brought V4332 Sgr in 1994 from anonymous quiescence at $V \sim 17$ to $V \sim 8.4$ mag at maximum, developping an M-giant spectrum that progressed toward later spectral types along the outburst evolution, with significant emission in the Balmer lines and [OI], [FeII], FeI, MgI and NaI but without marked P-Cyg type profiles (Martini et al. 1999). V838 Mon shares common characteristics with both such events, but some differences remain. First, both M 31-RedVar and V4332 Sgr had single-peaked and smooth light-curves, while V838 Mon experienced three maxima of widely different photometric and spectroscopic characteristics. The emission limited to only the Balmer lines resembles M 31-RedVar but the $M_{V}=-4.35$ peak brightness is much closer to V4332 Sgr. The presence and variability of P-Cyg profiles in V838 Mon match what saw in M 31-RedVar, while the $\sim \mathrm{F}$ dwarf progenitor is similar to the $\sim \mathrm{K}$ dwarf visible progenitor of V4332 Sgr. It could be that these three events are manifestations of the same and new class of astronomical objects.

\section{References}

Balick, B., Wilson, J., \& Hajian, A. R. 2001, ApJ, 121, 354

Blöcker, T., \& Schönberner, D. 1997, A\&A, 324, 991

Brown, N. J. 2002, IAU Circ., 7785

Drilling, J. S., \& Landolt, A. U. 2000, in Allen's Astrophysical Quantities, ed. A. N. Cox (AIP Press - Springer), 381

Iben, I. Jr, \& Tutukov, A. V. 1992, ApJ, 389, 369

Henden, A., Munari, U., \& Schwartz, M. B. 2002, IAU Circ. 7859

Kipper, T. 2001, Balt. Astron., 10, 403

Martini, P., Wagner, R., Tomaney, A., et al. 1999, AJ, 118, 1034

Mould, J., Cohen, J., Graham, J. R., et al. 1990, ApJ, 353, L35

Munari, U., \& Zwitter, T. 1997, A\&A, 318, 269

Rich, R. M., Mould, J., Picard, A., et al. 1989, ApJ, 341, L51

Serkowski, K., Mathewson, D. S., \& Ford, V. L. 1975, ApJ, 196, 261

Zwitter, T., \& Munari, U. 2002, IAU Circ., 7812 